\title{
ULTRAFAST OPTICAL STUDIES OF SURFACE REACTION PROCESSES AT SEMICONDUCTOR INTERFACES
}

\author{
Progress Report
}

for Period June 1, 1991 to May 31, 1992

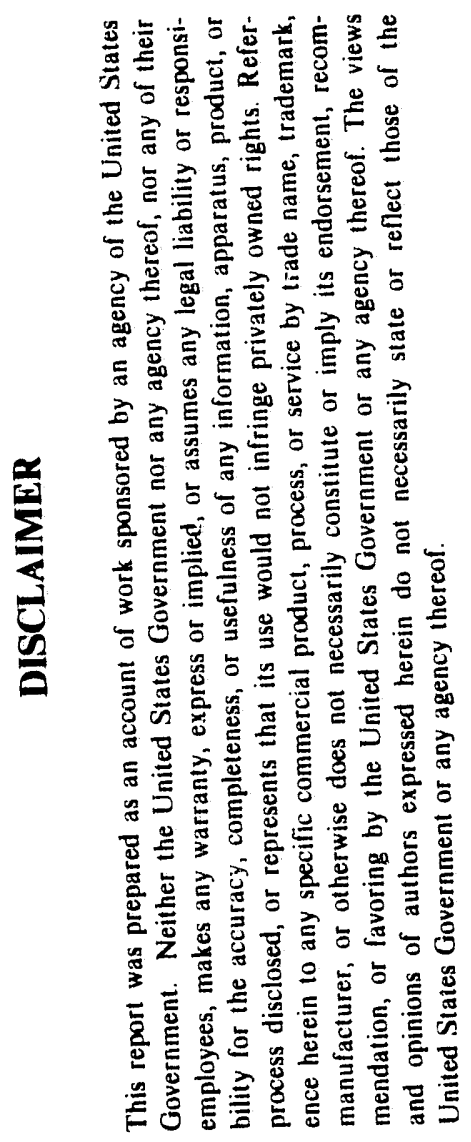

\author{
P.I.: R. J. Dwayne Miller \\ Department of Chemistry and Institute of Optics \\ University of Rochester \\ Rochester, NY 146?7
}

November 1991

Prepared for

THE U. S. DEPARTMENT OF ENERGY

AGREEMENT NO. DE-FG02- $\frac{9}{41} \frac{14185}{148}$ 
NOTICE

This rept was prepared as an account of work sponsored iy the United States Government. Neither the United States nor the Department of Energy, nor any of their emplnyees, nor any of their contractors, subcontractors, or their employees, makes any warranty, express or implied, or assumes any legal liability or responsibility for the accuracy, completeness, or usefulness of any information, apparatus, product or process disclosed or represents that its use would not infringe privately-owned rights. 


\begin{abstract}
Electron transfer processes at interfaces constitute a large and important class of surface reactions. In addition, the rectifying properties of semiconductor interfaces makes these systems one of the simplest and most efficient systems for converting and storing optical energy. However, the primary events of interfacial electron or hole carrier transfer and competing nonradiative (energy loss) channels are not well understood at surfaces. This research has explored the use of two novel optical techniques, Surface Space Charge Electro-optic Sampling and Surface Restricted Transient Grating Spectroscopy, to obtain the time evolution of the surface spatial distribution of photogenerated charge carriers and the photocarrier population dynamics at semiconductor interiaces, respectively. These studies have shown that carriers arrive at GaAs( 100 surfaces on the hundred feritosecond time scale and subsequently undergo interfacial charge transfer on the picosecond time scale to appropriate acceptors. These dynamics are competitive with carrier thermalization processes and support the concept that it may be possible to significantly improve energy conversion efficiencies by ninimizing energy loss through carrier thermalization. The exact dynamics still need to be resolved. A new laser system has been developed in the first year of funding that is capable of 10 femtosecond time resolution. This system will have sufficient time resolution to completely resolve both the electron and hole carrier non-radiative relaxation processes as well as the interfacial carrier charge transfer dynamics.
\end{abstract}




\section{Brogress Repolt}

One of the most important processes in surface mediated chemical reactions is the primary interfacial electron transfer step. Principally, there are two models developed for this process at semiconductor surfaces that differ on the energetic state of the electron as it crosses the surface barrier, i.e., thermalized vs. hot carrier injection models. The predicted dynamics for the two models differ by two orders of magnitude and are differentiable based on the observed time scale for interfacial electron transfer. Previous studies of interfacial transfer processes have been conducted on time scales which were not sufficient to follow the initial electron transfer dynamics. By taking advantage of the high quantum yields for electron transfer at semiconductor liquid junctions, we have been able to study the electron transfer process directly at working single crystal semiconductor surfaces using picosecond electro-optic sampling and transient grating techniques.

The transient grating method, in particular, has proven to be an extremely sensitive probe of semiconductor electron-hole carrier processes. The temporal evolution of the transient grating diffraction efficiency gives direct information on the minority carrier lifetime within the depletion layer which effectively measures interfacial electron or hole transfer processes along with the competing processes of surface state trapping. The temporal resolution is laser pulse width limited $\left(10^{-12}-10^{-13}\right.$ seconds). This method has turned out to be much more sensitive than originally proposed. We have demonstrated detection limits that correspond to $10^{-5}$ of a monolayer of reactive electron flux across the interface. This makes this method one of the most sensitive, if not the most sensitive, optical method developed to date for the study of surface processes. In addition, this new surface spectroscopy has introduced the concept of surface space charge field focusing to eliminate transport problems on the temporal resolution. The surface fields act to take the original spatial distribution of photocarriers and selectively drive the minority carrier to within tunneling distances of the interfacial acceptors on a 100 femtosecond time scale. This new effect provides surface selectivity and conserves the hundred femtosecond time resolution needed to follow all the carrier photophysical processes in the surface region. Our recent results on GaAs have demonstrated the field dependence on the carrier depletion dynamics at the surface and explicitly illustrated the space charge field focusing effect. This work was able to unambiguously follow the interfacial hole carrier depletion dynamics and found that the hole carrier interfacial charge transfer dyzamics to $\mathrm{Se}^{-2}$ acceptors was less than 30 picoseconds. This is a significant finding in that it is the fastest reported interfacial charge transfer process to date. The charge transfer dynamics appear to be operating close to the theoretical maximum, i.e., close to the rate limiting value for electron localization, as defined by the time scale for nuclear repolarization. 
The charge transfer dynamics determined in our grating studies are competitive with the time scales normally associated with carrier thermalization. These findings support Nozik's hypothesis that energy can be stored, in principle, through charge separation at a semiconductor liquid junction faster than the carriers can lose energy through non-radiative relaxation. The increase in energy conversion efficiency through the participation of hot carriers requires a quantitative determination of the carrier thermalization dynamics at a real interface (not UHV defect free surfaces) and the exact interfacial charge transfer kinetics. With this information, the branching ratio between hot carrier and thermalized carrier transfer can be determined. The thermalization dynamics in the presence of the defects are very likely to be accelerated over a defect free surface such that this measurement is the most crucial in determining the required dynamics for interfacial charge transfer in order to sustain a significant hot carrier component.

The above studies were limited in time resolution by the infrared probe pulse duration and not by intrinsic limitations in the method. It is only in the last two years that methods have been developed that enable the production of femtosecond infrared pulses. To this end, we have explored the combined use of frequency upconversion of the diffracted infrared signal pulse with a 100 femtosecond visible pulse. The summing process is analogous to a 100 femtosecond optical gate or boxcar that only measures the diffracted infrared intensity during the time interval of the summing pulse. In this manner, the time resolution has been improved to the 100 femtosecond regime (see Figure 1 for represc ntative data). This approach has the advantage of not having to tackle problems of pulse broadening in the infrared and producing a signal in the visible part of the spectrum which is much easier to detect. It has the disadvantage of turning a normal three pulse grating experiment into a five pulse experiment which is technically more demanding. Despite the extra complexity, this new approach has been brought on line in the first four months of the grant funding period. The next step in our surface grating studies will be to repeat our earlier work but with 300 times better time resolution. The current configuration should be able to completely resolve the issues surrounding photocarrier dynamics at interfaces.

The other line of study has been the development and application of Surfiace Space Charge Electro-optic Sampling as a means of following the spatial transport to the photogenerated carriers within the space charge region to the surface. This particular line of study has gained more importance since our discovery that the interfacial charge transfer dynamics are on the picosecond and potentially sub-picosecond time scale. It was originally assumed that the carrier transport to the surface would be several orders of magnitude faster than the interfacial kinetics. This is no longer true, and we will undoubtedly need to convolve the carrier depletion dynamics to the arrival times of the carrier distribution at the surface. To this end, we have directly determined the spatial transport of the carriers by following the change in surface field amplitude as the minority carriers 
are accelerated to the surface. The spatial distribution of the photocarriers can then be determined through Poisson's equation for field amplitudes.

In this line of study, we have determined the fundamental upper limit in the detection of electrical transients. We have demonstrated that the electro-optic effect has an effective electrical bandwidth of approximately 10 Terahertz. This corresponds to an effective time resolution of 30 femtoseconds in the detection of an electrical signal. In essence, we have developed the world's fastest optical oscilloscope. In the last few months, this work has shown that the hole carrier at $n$ $\mathrm{GaAs}$ interfaces arrives at the surface reaction plane in less than 200 femtoseconds (see Figure 2). Again, this time scale is faster than that expected for carrier thermalization. This work strongly supports the concept that the carriers arrive at the surface reaction plane hot, after space charge field acceleration, and are capable of transmission prior to thermalization for appropriate charge acceptors.

At present, the main questions concern the transport component of the electron. The effective mass of the electron is a factor of ten smaller than the hole carrier such the electron dynamics could approach the 10 femtosecond regime for transport across the surface space charge field. If correct, the electron carrier could be made to propagate essentially ballistically (no scattering, collisions, or thermalization) to the surface in a p-type semiconductor. This could provide important insight into proper design considerations for electron based acceptors and yet even faster interfacial charge transfer. We have previously been limited in our time resolution by the approximately 200 femtosecond pulses of our laser system in these studies; The first year of funding has seen the build up of a Titanium:Sapphire laser system which produces directly 50 femtosecond pulses in the near infrared. The system was installed in July. Since then, a ne'el regenerative pulse amplifier has been constructed and the system is being further compressed down to 10 femtoseconds. With this new laser systems, we will be able to pull out even the electron transport dynamics. This system will also be invaluable in completely following the carrier thermalization and charge transfer processes in our surface grating work.

The above studies have highlighted that last six months progress in following p.eviously established research paths. The research will be modified to take advantage of recent research capabilities made available through collaborations with Professor Yong Li Go in the Department of Physics. A time of flight electron spectrometer has been built up by Professor Go's group, and we will have access to an ultrahigh vacuum surface machine. The above mentioned Titanium:Sapphire laser system has opened up the possibility of doing femtosecond surface photoemission. This spectroscopic probe will provide a nice compliment to the optical studies with the distinct advantage that this method will directly determine the energetic distribution of the electrons (and potentially hole carriers) as they relax. Thus, not only will we have dynamics we will also know the time dependent energetics of the electron distribution at the surface. This is an important 
devclopment as the electron energetics do not relax in a monotonic fashion. The complete information on the carrier energetics that this approach provides will be crucial in developing a detailed model of the electron transfer processes at surfaces.

The combined use of these three spectroscopies have been able to map out the interfacial carrier transfer dynamics, the carrier spatial transport, and with the implementation of timeresolved photoemission, the energetics. The studies are progressing to the point that quantitative numbers can be put on these variables. This is the main information needed to characterize the fundamental electronic coupling between molecular states at the surface and the extended electronic states of the solid state. This dynamical information will ultimately need to be understood in terms of the structure of the interface which will require other approaches. 


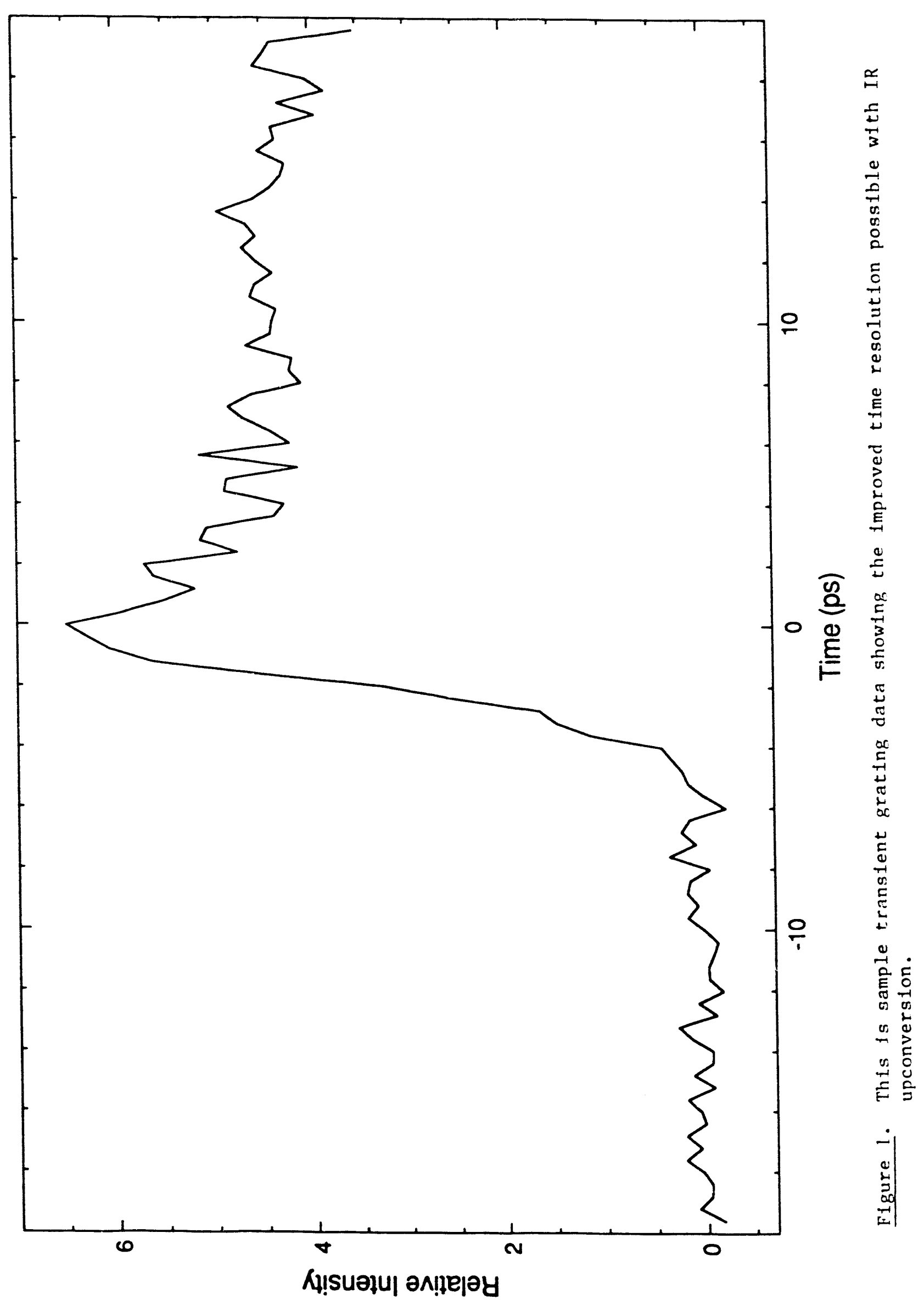


Experimental Results from Reflection Electro-optic Sampling Studies of n-GaAs (100)/oxide Interface

a) Polarization dependence

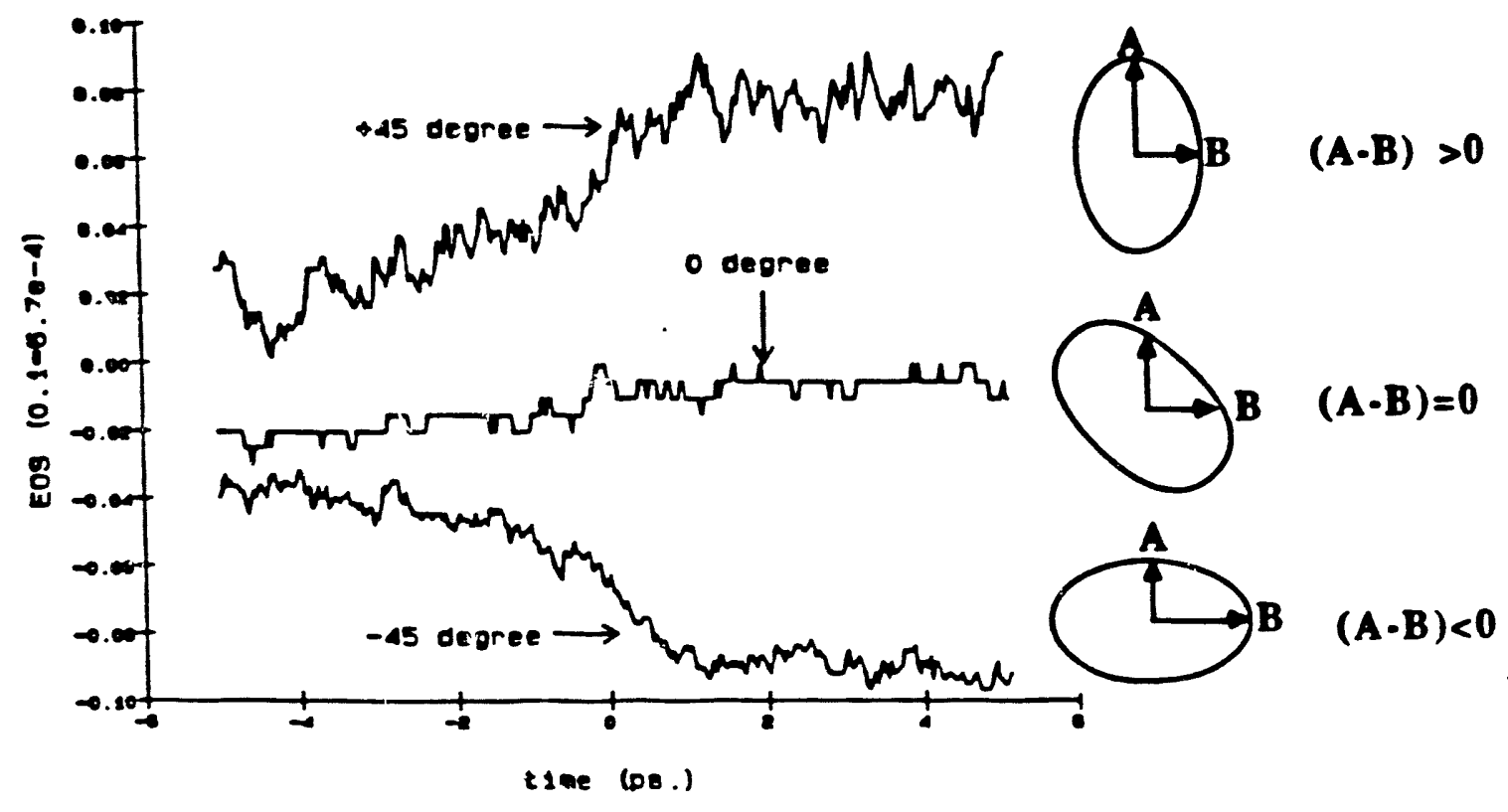

demonstrates signal due to electro-optic effect

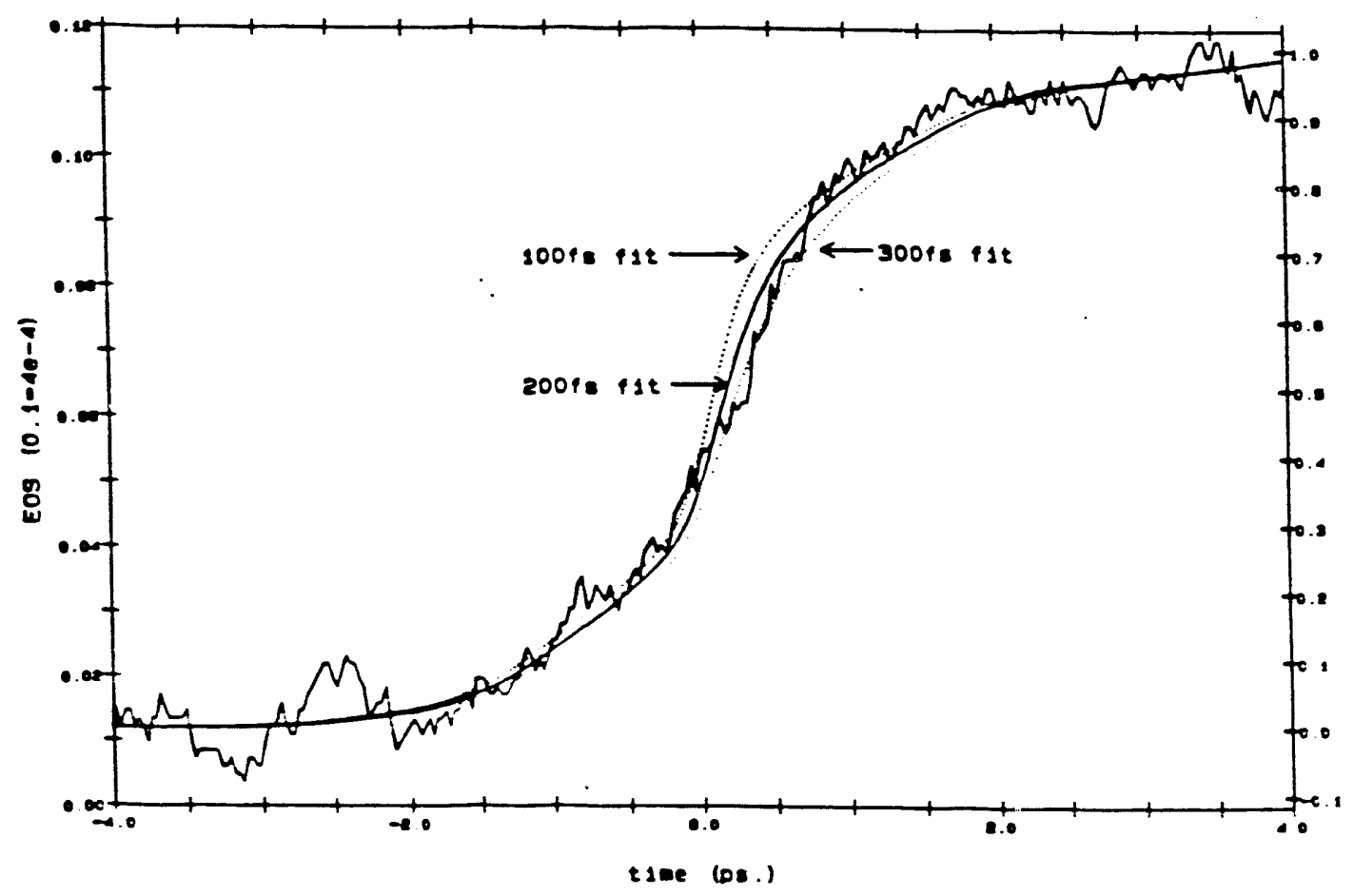

$200 \mathrm{fs}$ component due to hole carrier motion to the surface. 

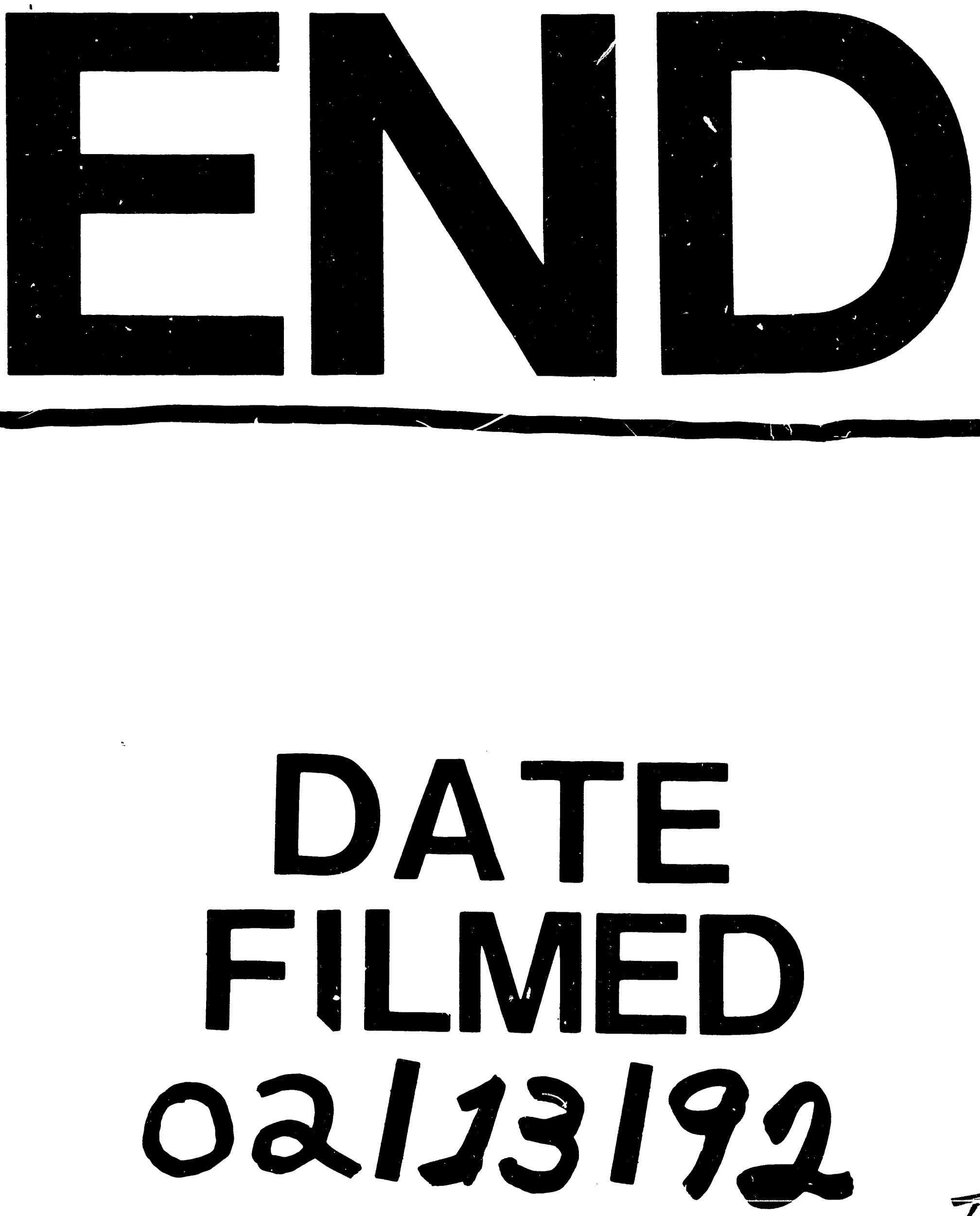

$I$ 
